\section{Giant Intraductal Papilloma: A Rare Case}

Keywords: Intraductal papilloma; Mammography; Breast ultrasound; Breast magnetic resonance imaging

\begin{abstract}
Intraductal papilloma is a benign fibroepithelial tumor of the breast. It constitutes approximately $5 \%$ of all breast cancers. Although it can be seen at any age, it is mostly seen in late reproductive age and during perimenopause (40-50 years). However, giant intraductal papillomas are very rarely seen. In this paper, we aimed to present a 45-year-old female patient who underwent multimodality imaging and had pathological results but in whom the distinction between benign and malignant tumors could not be made clearly by imaging findings.
\end{abstract}

\section{Introduction}

Intraductal papillomas are usually detected incidentally on mammography. They are divided into two subtypes as central and peripheral. In the central subtype, they are usually $2-3 \mathrm{~mm}$ in size including below $1 \mathrm{~cm}$. In the peripheral subtype, they are usually multiple and can reach a large size [1,2]. Giant intraductal papillomas are very rarely seen. Only 8 case reports were documented in the literature [3]. We think that our case is the 9th case in the literature. As in our case, giant intraductal papillomas are not usually considered as preliminary diagnosis in the first step because they are rarely seen in giant size and do not have typical imaging findings on Mammography (MG), Ultrasound (US), and breast Magnetic Resonance İmaging (MRI) [3]. Since the distinction between benign and malignant tumors cannot be made clearly by imaging findings in general, the precise diagnosis in these cases is made after the surgery.

\section{Case Report}

A 45-year-old female patient was admitted to our clinic with complaints of a bruise on the skin and an increased mass in the left breast. For this reason, the patient underwent MG and ultrasound. On MG, a nodular high-density lesion with smooth contour (approximately $6.5 \times 6 \mathrm{~cm}$ in size) was detected in both the upper middle quadrant and the retroareolar region of the left breast (Figure 1a). On ultrasound, a semisolid-cystic mass (approximately $6.5 \times 6 \mathrm{~cm}$ in size), which contained a solid mural, hypervascular component $(24 \times 28$ $\mathrm{mm}$ in size) and rendered papillary protrusions into the cyst, was observed in the posterior retroareolar region of the left breast (Figure 1b). The patient underwent dynamic breast MRI because it contained a solid vascular area. On T2-weighted images, a hemorrhagic cystic lesion (6 $\mathrm{cm}$ in size), which led to fluid-fluid level and could not be suppressed in fat-suppressed sequences, was detected. A solid mural component with irregular border $(4 \times 2 \mathrm{~cm}$ in size), which appeared hypointense on T1-weighted images and mild hyperintense on T2Aweighted images and which showed a contrast in early phase and type 3 contrast patern in late phase following İntravenous Contrast Agent (IVCA) administration, was found in the posterior basal segment of the cystic lesion (Figures $1 \mathrm{c}$ and $1 \mathrm{~d}$ ). The patient was classified as BI-RADS $4 \mathrm{a}$ based on the current imaging findings. Then, tru-cut

\section{Journal of}

Clinical \& Medical Case Reports

\author{
Ayşegül Altunkeser ${ }^{1 *}$, Ender Alkan ${ }^{1}$, Mehmet Ali \\ Eryılmaz $^{2}$, Süleyman Bakdık ${ }^{3}$ and Yaşar Ünlü ${ }^{4}$ \\ 'Department of Radiology, University of Health Sciences, Konya Edu- \\ cational and Research Hospital, Konya, Turkey \\ 'Department of General Surgery, University of Health Sciences, Konya \\ Educational and Research Hospital, Konya, Turkey \\ ${ }^{3}$ Department of Radiology, Meram Medical Faculty, Necmettin Er- \\ bakan University, Konya, Turkey \\ ${ }^{4}$ Department of Pathology, University of Health Sciences, Konya Edu- \\ cational and Research Hospital, Konya, Turkey \\ Address for Correspondence: \\ Ayşegül Altunkeser, Department of Radiology, University of Health \\ Sciences, Konya Educational and Research Hospital, Konya, Turkey, \\ Tel: +90 33222100 00; E-mail: aaltunkeser@hotmail.com \\ Submission: 01 April, 2017 \\ Accepted: 29 April, 2017 \\ Published: 05 May, 2017 \\ Copyright: () 2017 Altunkeser A. This is an open access article \\ distributed under the Creative Commons Attribution License, which \\ permits unrestricted use, distribution, and reproduction in any medium, \\ provided the original work is properly cited.
}

biopsy was performed under ultrasound guidance. The lesion that was histopathologically defined as an intraductal papillary lesion was removed by excisional biopsy. Similarly, pathology result revealed an intraductal papillary tumor (Figure 2).

\section{Discussion}

Intraductal papillomas are frequently seen during the perimenopausal period. With the increased use of breast ultrasound, it is also increasingly seen in young asymptomatic patients. If solitary intraductal papillomas are symptomatic, they can lead to some

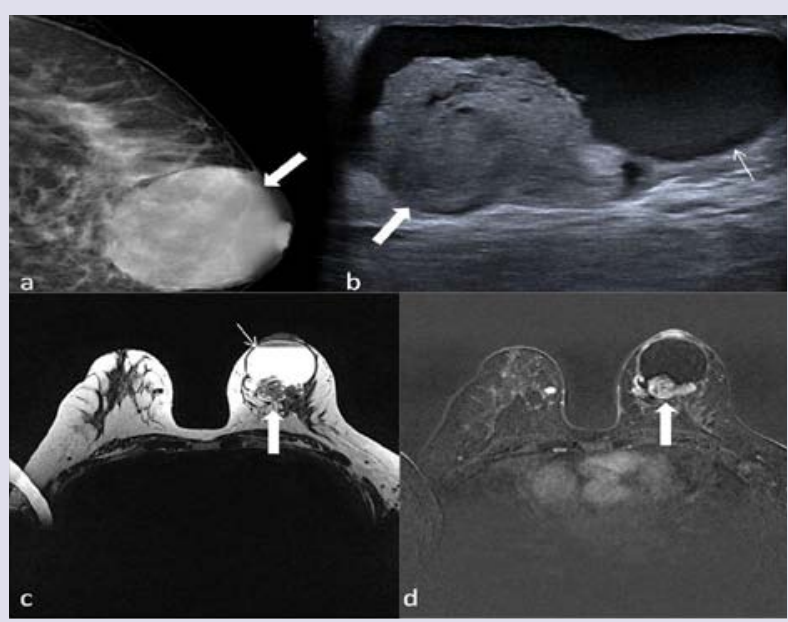

Figure 1: (a) Left breast mammography craniocaudal (CC), views a large well defined, dense mass is observed (arrow). (b) B-mode ultrasound reveals a large intracystic (thin arrow) solid lesion (thick arrow). (c) On the axial T2Aweighted image, the intracystic solid component is seen as hypointense (thick arrow). A fluid level is also present (thin arrow). (d) On the axial T1A-weighted contrast enhanced image, it is seen that the large intracystic solid component shows significant contrast enhancement (arrow). 


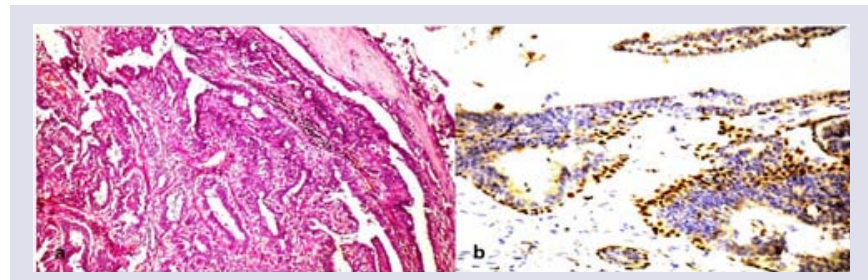

Figure 2: On pathologic examination. (a) Papillomatous formation that shows papillary extensions towards the lumen within the dilated duct. Hex100. (b) The presence of myoepithelial cells with p63-positive expression in papillary structures. p63×150.

complaints such as bloody nipple discharge or nipple displacement [4]. Our patient was 45 years old, and was in the perimenopausal period. The patient's complaints were a palpable mass and a bruise on the skin. Our patient had no bloody nipple discharge or nipple displacement.

Imaging findings for intraductal papillomas are nonspecific, and show a broad spectrum. Due to the nonspecific imaging findings, the distinction between benign and malignant tumors cannot be made clearly, and tissue sampling is required [2]. While intraductal papillomas present as a nonspecific density on mammography, they are seen as an intracystic solid mass filling the duct on ultrasound [2,5]. In our case, mammography revealed a high-density well defined mass, and ultrasound revealed a complex cystic lesion with marked vascularity.

There are not many sources describing MRI characteristics of intraductal papillomas. While large intraductal papillomas can be seen as contrasting nodules with or without intraductal component, small intraductal papillomas can remain hidden. They show wash out or plateau kinetics, and are difficult to differentiate from malignant lesions [6]. They may show restricted diffusion on Diffusion-Weighted İmages (DWI) depending on the amount of cells as in malignant tumors $[7,8]$. In our case, MRI revealed a large semisolid mass which had type 3 contrast pattern and showed restricted diffusion. The lesion was classified as BI-RADS $4 \mathrm{a}$ based on the multimodality imaging findings.

Because giant intraductal papillomas do not have specific imaging findings, tru-cut biopsy should be performed under ultrasound guidance in order to exclude malignancy [3]. In our patient, ultrasound-guided tru-cut biopsy was reported as an intraductal papillary lesion. Some studies have reported that precancerous or neoplastic lesions were present in up to $80 \%$ of patients with multiple intraductal papillomas and that the risk of breast cancer was higher in these patients compared to the general population [4,9]. Although total mastectomy has been reported in giant intraductal papillomas, the standard treatment is excision [3,10]. Our patient underwent excisional biopsy because tru-cut biopsy did not represent the whole tissue, the radio-pathological discordance was present, and potentially precancerous lesions may accompany. Finally, pathology result revealed an intraductal papillary tumor.

\section{Results}

The imaging features in giant intraductal papillomas are nonspecific as they are in small intraductal papillomas. They can mimic malignant neoplasms with their diffusion and contrast patterns especially on MRI. Preoperative biopsy is recommended for a definite diagnosis. However, even if the result is benign, excision is recommended due to accompanying premalignant lesions.

\section{References}

1. Lewis JT, Hartmann LC, Vierkant RA, Maloney SD, Pankratz VS, et al (2006) An analysis of breast cancer risk in women with single, multiple, and atypical papilloma. Am J Surg Pathol 30: 665-672.

2. Eiada R, Chong J, Kulkarni S, Goldberg F, Muradali D (2012) Papillary lesions of the Breast: MRI, ultrasound, and mammographic appearances. AJR Am J Roentgenol 198: 264-271.

3. Fatemi Y, Hurley R, Grant C, Henrichsen T, Chen B, et al. (2015) Challenges in the management of giant intraductal breast papilloma. Clin Case Rep 3: 7-10.

4. Mulligan AM, O'Malley FP (2007) Papillary lesions of the breast: a review. Adv Anat Pathol 14: 108-119.

5. Ganesan S, Karthik G, Joshi M, Damodaran V (2006) Ultrasound spectrum in intraductal papillary neoplasms of breast. Br J Radiol 79: 843-849.

6. Daniel BL, Gardner RW, Birdwell RL, Nowels KW, Johnson D (2003) Magnetic resonance imaging of intraductal papilloma of the breast. Magn Reson Imaging 21: 887-892.

7. Woodhams R, Matsunaga K, Kan S, Hata H, Ozaki M, et al. (2005) ADC mapping of benign and malignant breast tumors. Magn Reson Med Sci 4: 35-42.

8. Rubesova E, Grell AS, De Maertelaer V, Metens T, Chao SL, et al. (2006) Quantitative diffusion imaging in breast cancer: a clinical prospective study. J Magn Reson Imaging 24: 319-324.

9. Carter D (1977) Intraductal papillary tumors of the breast: a study of 78 cases. Cancer 39: 1689-1692.

10. Farid MK, Sarma HN, Ramesh K, al Fituri O, Visveswara RN (1997) Giant juvenile papillomatosis of the breast: report of two cases. East Afr Med J 74: 116-117. 